\title{
Multi-layer composite mechanical modeling for the inhomogeneous biofilm mechanical behavior
}

\author{
Xiaoling Wang*,†, \\ Mudong Hao* \\ ${ }^{*}$ School of Mechanical Engineering, University of Science and \\ Technology Beijing, Beijing 100083, P. R. China \\ ${ }^{\dagger}$ Harvard John A. Paulson School of Engineering and Applied Sciences \\ Faculty of Arts and Sciences Harvard University \\ Cambridge MA 02138, USA \\ Laboratory of Nonlinear Mechanics (LNM) \\ Institute of Mechanics, Chinese Academy of Sciences \\ 15, Bei Si Huan Xi Lu, Beijing 100190, China \\ $\S_{x i a o l i n g @ m e . u s t b . e d u . c n}$
}

Received 24 September 2015

Revised 31 December 2015

Accepted 24 February 2016

Published 27 April 2016

\begin{abstract}
Experiments showed that bacterial biofilms are heterogeneous, for example, the density, the diffusion coefficient, and mechanical properties of the biofilm are different along the biofilm thickness. In this paper, we establish a multi-layer composite model to describe the biofilm mechanical inhomogeneity based on unified multiple-component cellular automaton (UMCCA) model. By using our model, we develop finite element simulation procedure for biofilm tension experiment. The failure limit and biofilm extension displacement obtained from our model agree well with experimental measurements. This method provides an alternative theory to study the mechanical inhomogeneity in biological materials.
\end{abstract}

Keywords: Biofilm; finite element method; multi-layer composite mechanical model; UMCCA; inhomogeneity.

\section{Introduction}

Biofilms are regarded as communities of tightly associated bacteria encased in an extracellular matrix (ECM) found on almost all kinds of surfaces. ${ }^{1}$ Biofilms not only have many potential applications, but also can cause problems. For example, biofilm can be utilized in industrial wastewater treatment for absorbing heavy metals, ${ }^{2}$ it also can be used to purify the eutrophic water by denitrification of bacterial cells, ${ }^{3}$ and likewise, biofilm is used in microbial fuel cells to provide clean energy. ${ }^{4,5}$

\$Corresponding author. 
However, biofilm can cause many kinds of problems, such as infection of wounds, contamination of implants and medical apparatus, and metal corrosion ${ }^{6}$; furthermore, it can induce drug resistance because of biofilm attachment to some organs in our body. ${ }^{7,8}$ Accordingly, biofilm attracts researchers from multi-disciplinary fields, such as physics, biology, material science, and mechanics. ${ }^{9,10}$

Experiments showed that biofilm is inhomogeneous in density ${ }^{11}$ the diffusion coefficient, ${ }^{12}$ morphology, ${ }^{13}$ and phenotypes. ${ }^{14}$ Xiaoling ${ }^{22}$ shows that the B. subtilis biofilm thickness is different along radius, with the high thickness region corresponding to wrinkles. The experiment by Wilking et al. ${ }^{13}$ shows that wrinkles distributed in the biofilm can facilitate nutrient and liquid transportation. Xiaoling's experiment $^{22}$ also shows the three phenotypes evolution during biofilm growth, and that different phenotypes are distributed heterogeneously in biofilm. Besides bacterial cells, another main composition of biofilm is extracellular polymeric substances (EPS), which are composed primarily of extracellular polysaccharides with high molecular weight and high concentration; they together provide biofilm structure integrity. ${ }^{15}$ Ohashi et al. ${ }^{16}$ showed that the elasticity coefficient is positive correlation with biofilm density, which approves mechanical inhomogeneity of biofilm.

There are few theoretical models to explain the biofilm inhomogeneity. Beyenal and Lewandowski ${ }^{12}$ suggested biofilm was composed of layers with different diffusion coefficients. Laspidou and Rittmann ${ }^{11,18}$ and Laspidou et al. ${ }^{17}$ developed the unified multiple-component cellular automaton (UMCCA) model, in which they assumed biofilm was made up of three solid species and four soluble substrates; they obtained all these components' distributions and density distribution along the biofilm thickness, which showed that the density decreases from the bottom to the top along the biofilm thickness. The high heterogeneities of both components and the density in biofilm can cause mechanical inhomogeneity, but the existing models remain lacking.

In our paper, we assume the biofilm is made up of three solid species, which are inert biomass, active bacteria, and EPS. Our objective is to establish a multi-layer composite model based on UMCCA model to describe biofilm mechanical behavior. We determine the unknown parameters in our model from experiment measurements, and from this we obtain Young's modulus distribution along the biofilm thickness. Our model is further used to simulate biofilm tension experiment, and the results agree well with experimental measurements.

\section{Theoretical Model}

\subsection{Composite mechanical model}

In materials science, a composite material is made of two or more than two materials that are mixed in physical or chemical ways. We assume biofilm is a composite material and made up of inert biomass, active bacteria, and EPS, which play main roles to biofilm mechanical property. Since there is a density inhomogeneity along biofilm thickness, we divide biofilm into $N$ layers uniformly, and each layer is assumed to be a composite material, which is uniform, isotropic, and elastic. Each 
component of biofilm has its own Young's modulus and contributes to composite Young's modulus of biofilm.

The composite Young's modulus can be calculated in the view of composite elasticity theory, which was proposed by Willis. ${ }^{19}$ Let $E_{i}(i=1,2,3)$ indicate the Young's moduli of three components, and let $\nu_{i}(i=1,2,3)$ denote the Poisson's ratios of the three components. Here, $i=1$ indicates the inert biomass, $i=2$ indicates the active biomass, and $i=3$ indicates the EPS. We obtain shear modulus $\mu$ and bulk modulus $\kappa$ of each component from Eq. (1).

$$
\mu_{i}=\frac{E_{i}}{2\left(1+\nu_{i}\right)}, \quad \kappa_{i}=\frac{\nu_{i} \cdot E_{i}}{\left(1+\nu_{i}\right)\left(1-2 \nu_{i}\right)} .
$$

We deduce the composite shear modulus of biofilm from Willis ${ }^{19}$ theory:

$$
\mu_{\text {comp }}=\left\{\sum_{i=1}^{3} c_{i} \frac{5 \hat{\mu}(3 \hat{\kappa}+4 \hat{\mu})}{6 \mu_{i}(\hat{\kappa}+2 \hat{\mu})+\hat{\mu}(9 \hat{\kappa}+8 \hat{\mu})}\right\}^{-1} \sum_{i=1}^{3} c_{i} \frac{5 \hat{\mu}(3 \hat{\kappa}+4 \hat{\mu}) \mu_{i}}{6 \mu_{i}(\hat{\kappa}+2 \hat{\mu})+\hat{\mu}(9 \hat{\kappa}+8 \hat{\mu})} .
$$

where $\hat{\mu}$ and $\hat{\kappa}$ are shear and bulk moduli of a certain component (parent phase), which distributes more uniformly in the composite material than the other components. In biofilm, EPS is more homogenous and has less changes along biofilm thickness compared with the other two components. Therefore, $\hat{\mu}=\mu_{3}, \hat{\kappa}=\kappa_{3} ; c_{i}$ is the mass fraction of each component among the total mass of three components and it can be expressed as

$$
c_{1}=\frac{X_{\mathrm{res}}}{X_{a}+X_{\mathrm{res}}+X_{\mathrm{EPS}}}, \quad c_{2}=\frac{X_{a}}{X_{a}+X_{\mathrm{res}}+X_{\mathrm{EPS}}}, \quad c_{3}=\frac{X_{\mathrm{EPS}}}{X_{a}+X_{\mathrm{res}}+X_{\mathrm{EPS}}} .
$$

where the $X_{\text {res }}, X_{a}$, and $X_{\mathrm{EPS}}$ are concentrations of inert biomass, active bacteria, and EPS in one layer, respectively. The composite Young's modulus of each layer can be obtained from Eq. (4):

$$
E_{\text {comp }}=2 \mu_{\text {comp }}\left(1+\nu_{\text {comp }}\right) \text {. }
$$

where the composite Poisson's ratio $\nu$ should be equal to 0.3 since the $\nu$ values of solid components are all equal to 0.3 . The composite Young's modulus $E_{\text {comp }}$ can show the stress-strain properties of elastic composite material. ${ }^{17,19}$

\subsection{Parameter determination}

We determine all unknown values of our model from experimental analysis. In our composite biofilm model, we divide the biofilm with $0.25 \mathrm{~mm}$ thickness into 10 layers, the thickness of each layer is $0.025 \mathrm{~mm}$. We consider that the young biofilm grows within 24.5 days, ${ }^{11}$ and assume biofilm is homogenous in each layer; each layer has its own density and mechanical properties.

Firstly, we calculate shear modulus $u_{i}$ and bulk modulus $\kappa_{i}$ of each component from Eq. (1), in which $E_{\mathrm{res}}=240 \mathrm{~Pa}, E_{a}=10 \mathrm{~Pa}, E_{\mathrm{EPS}}=60 \mathrm{~Pa}$ from the works of 
X. Wang et al.

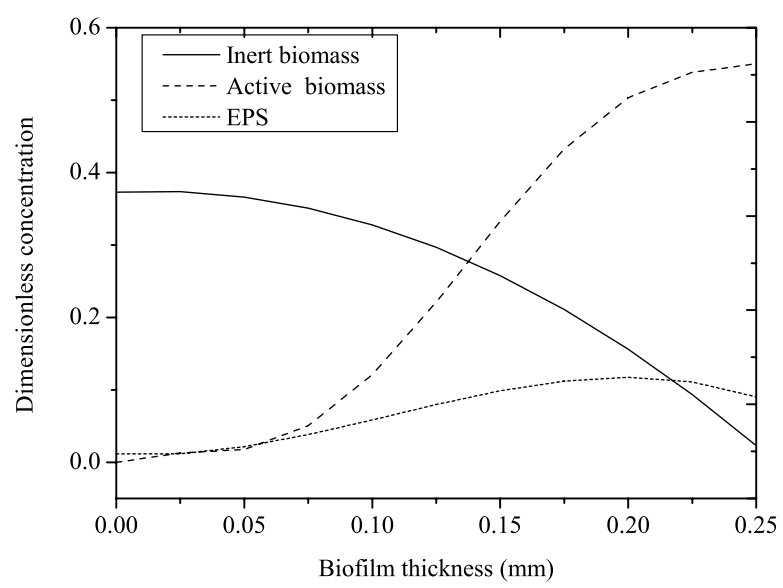

Fig. 1. The concentration curves of three solid components.

Stoodley et $a .^{20}$ and Picioreanu et $a l .{ }^{21}$

$$
\begin{array}{ll}
\mu_{1}=92.301 \mathrm{~Pa}, \quad \kappa_{1}=138.462 \mathrm{~Pa} \\
\mu_{2}=3.846 \mathrm{~Pa}, \quad \kappa_{2}=5.769 \mathrm{~Pa} \\
\mu_{3}=23.077 \mathrm{~Pa}, \quad \kappa_{3}=34.615 \mathrm{~Pa} .
\end{array}
$$

We can get composite shear modulus from Eq. (2):

$$
\mu_{\text {comp }}=\left(0.403 c_{1}+1.7 c_{2}+c_{3}\right)^{-1}\left(37.2 c_{1}+6.54 c_{2}+23.007 c_{3}\right) .
$$

Secondly, we determine the mass fraction of each component $c_{i}$ in Eq. (5). We can acquire them from each component concentration curve in the work of Laspidou et al. ${ }^{17}$ as shown in Fig. 1. Then we can obtain $c_{i}$ values from Eqs. (3) and (6)-(8).

The concentration formulas of three solid components are as follows:

$$
\begin{gathered}
X_{\text {res }}=-6.3217 x^{2}+0.1804 x+0.3730, \\
X_{a}=6400 x^{5}-4358 x^{4}+939.72 x^{3}-59.822 x^{2}+1.5214 x+0.0009, \\
X_{\mathrm{EPS}}=-32.448 x^{3}+10.331 x^{2}-0.2408+0.0117,
\end{gathered}
$$

Finally, we can obtain composite shear modulus and Young's modulus from Eqs. (4) and (5).

\section{Finite Element Simulation and Discussion}

\subsection{Finite element model}

We simulate the biofilm tension based on our composite mechanical model under loading conditions from Ohashi et al.'s experiment. ${ }^{16}$ In the experiment, the biofilm was cultured on the outer surface of the two non-connected but adjacent polyethylene tubes, the outer diameter of each tube is $4.76 \mathrm{~mm}$. The experiment is schematically shown in Fig. 2. The biofilm extension displacement between tubes, and 
the loading force on biofilm were measured. The failure stress in biofilm was obtained from Eq. (9).

$$
\sigma=\frac{F}{A_{b}}=\frac{F}{\left(\delta^{2}+d \delta\right) \pi} .
$$

where $\delta$ is the biofilm thickness, $d$ is the outer diameter of polyethylene tubes, and $A_{b}$ is the biofilm cross-sectional area.

Two main results from experiment are obtained: one is that the elastic failure stress of a mature biofilm is in the range of $500-1000 \mathrm{~Pa}$, and another is that the measured extension displacements of 11-days and 29-days old biofilm are 4.5 and $2 \mathrm{~mm}$, respectively, as shown in Fig. 3 .

We establish a two-dimensional finite element model during biofilm extension, as shown in Fig. 4. The two thick black lines in the bottom represent polyethylene tubes in the experiment, the gap between these two tubes is represented by white region between two thick black lines, and the meshed region means biofilm grows on tubes. We consider two biofilm models in our simulation: one is a composite biofilm model composed of different layers with different Young's moduli, and the other is a uniform biofilm model with an average Young's modulus of 73.208 Pa, by first integrating and then differentiating Young's modulus used in composite biofilm model. Both extension displacement and force loading conditions for two models are considered.

\subsection{Displacement loading}

In order to get failure stress, we assume that the extension displacement and biofilm age (11-29 days) have a linear relationship $\left|\left(l_{11}-l_{29}\right) /\left(t_{11}-t_{29}\right)\right|=\mid\left(l_{24.5}-\right.$ $\left.l_{29}\right) /\left(t_{24.5}-t_{29}\right) \mid$ from Fig. 3 and estimate the extension displacement of the 24.5days old biofilm as $2.625 \mathrm{~mm}$.

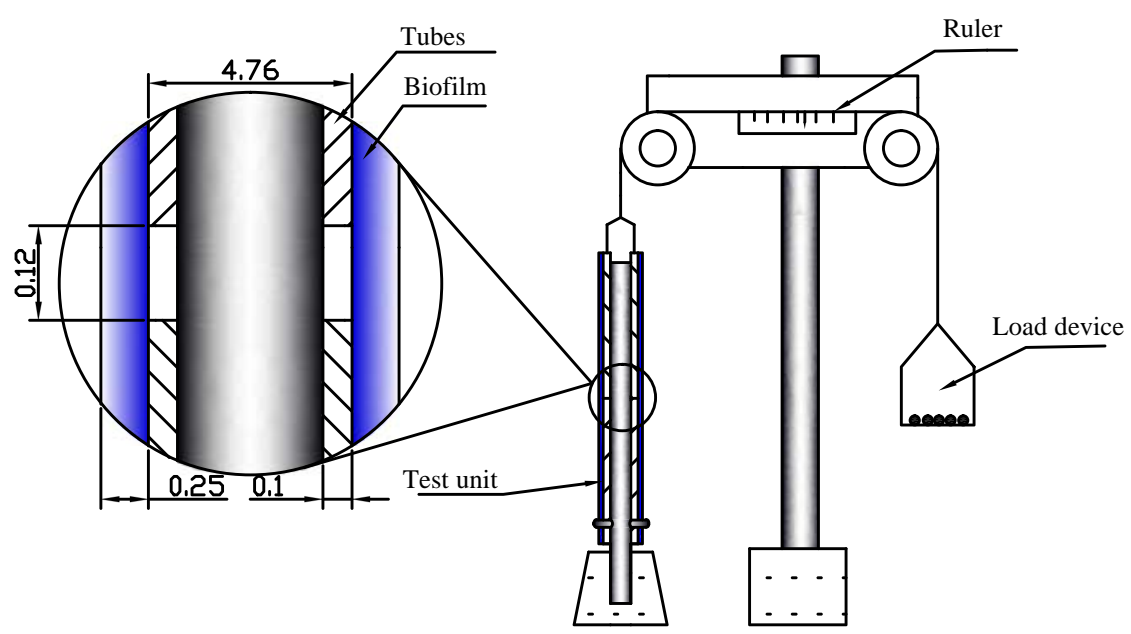

Fig. 2. Biofilm tension device (all units in $\mathrm{mm}$ ). 


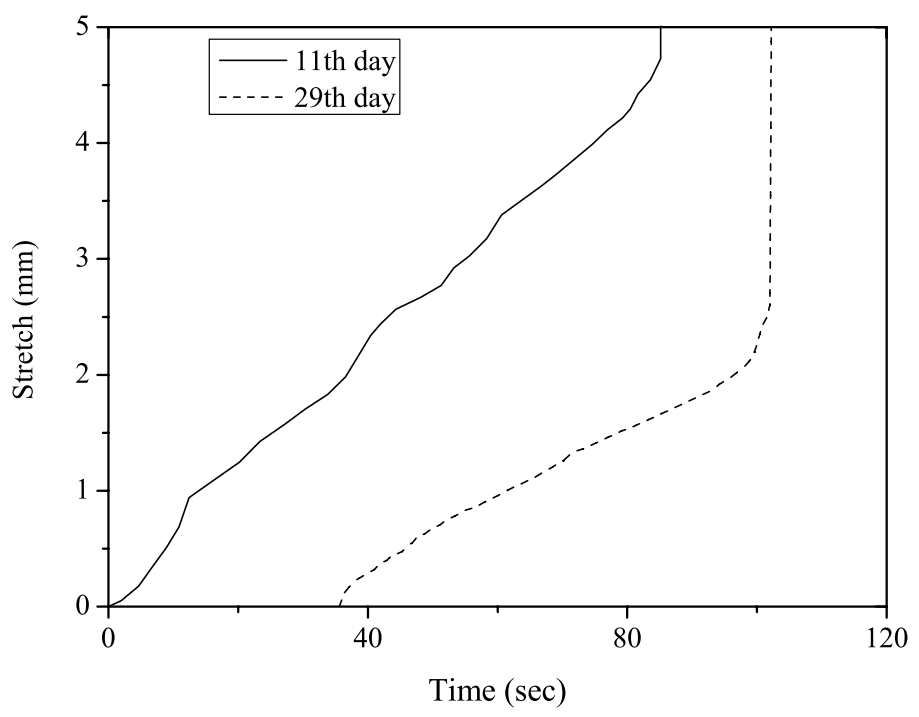

Fig. 3. Biofilm stretching on the 11 th and 29 th days. ${ }^{16}$

We set up the uniform and composite finite element biofilm models using ABAQUS. To compare with experimental measurements, we obtain the stress values along extension directions for both uniform and composite models, as shown in Fig. 5.

To compare with experimental measurements, we calculate the average tension stress in biofilm. We choose stresses along $Y$-axis from different regions of both models, and the six selected positions are shown in Fig. 6.

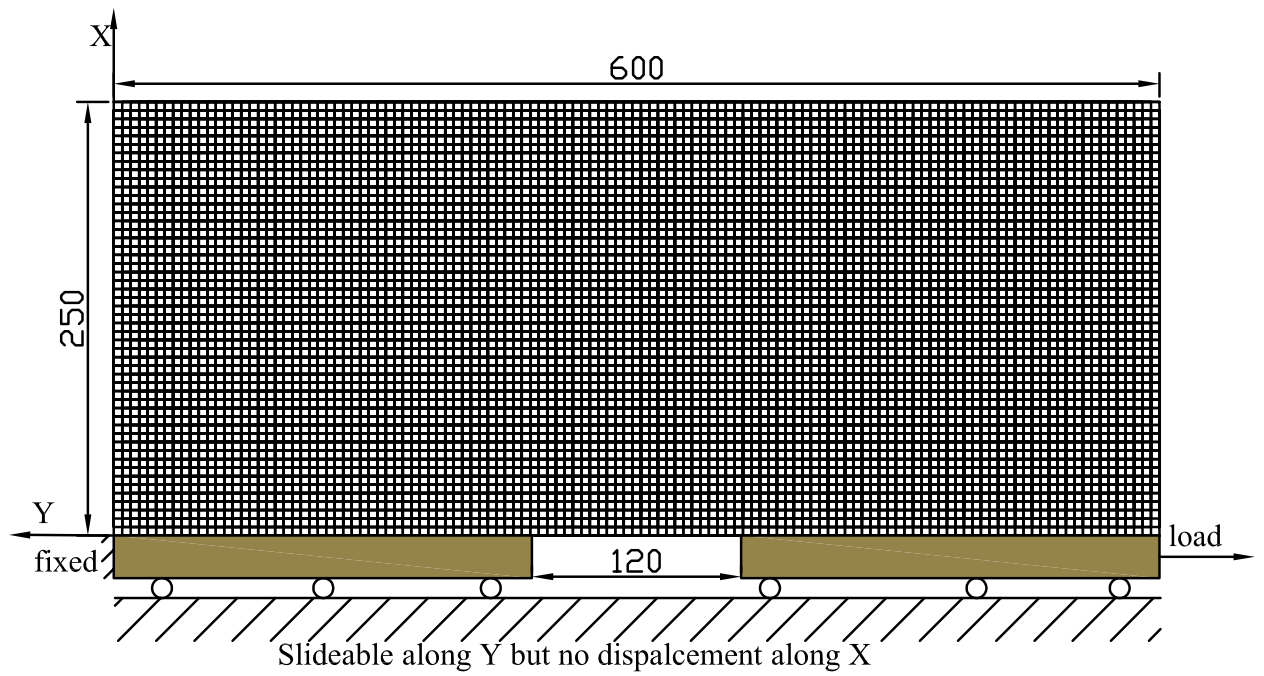

Fig. 4. Boundary conditions (all units in $\mu \mathrm{m}$ ), ${ }^{17}$ the negative $Y$-axis is loading direction. 


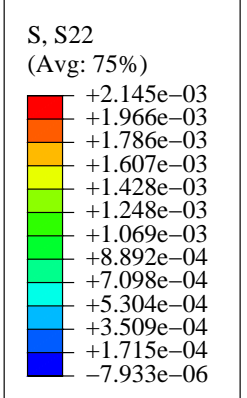

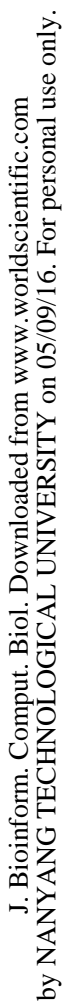

\section{S, S22 \\ (Avg: 75\%)}

$+4.812 \mathrm{e}-03$

$+4.408 \mathrm{e}-03$

$+4.003 \mathrm{e}-03$

$+3.598 \mathrm{e}-03$

$+3.193 \mathrm{e}-03$

$+2.789 \mathrm{e}-03$

$+2.384 \mathrm{e}-03$

$+1.979 \mathrm{e}-03$

$+1.574 \mathrm{e}-03$

$+1.169 \mathrm{e}-03$

$+7.647 \mathrm{e}-04$

$+3.599 \mathrm{e}-04$

$-4.484 \mathrm{e}-05$

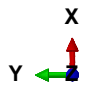

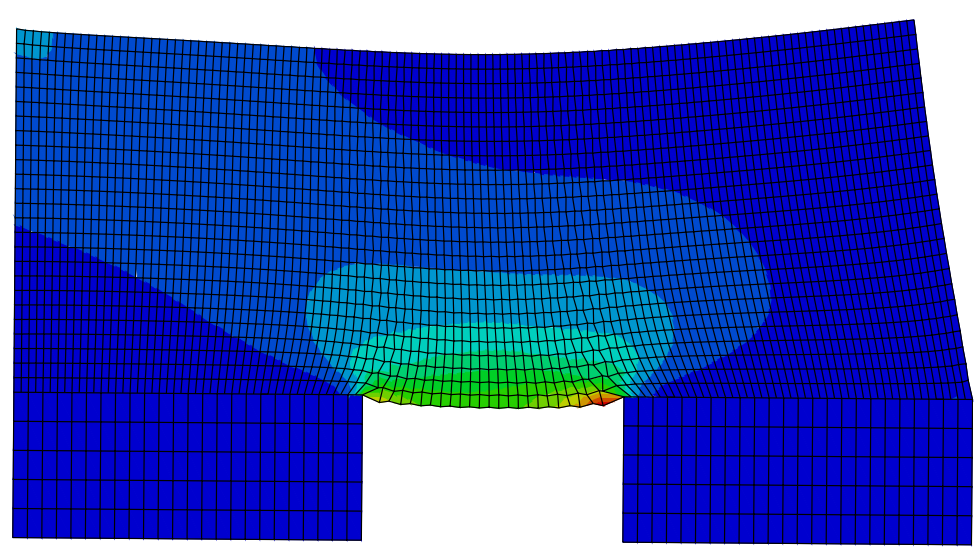

(a)

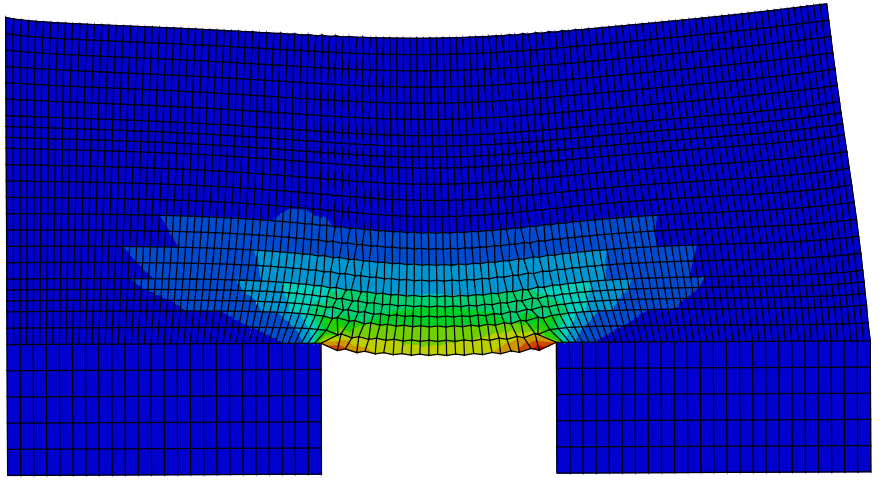

(b)

Fig. 5. The normal stress distribution (S22) along tension direction under displacement loading for (a) uniform and (b) composite biofilm models.

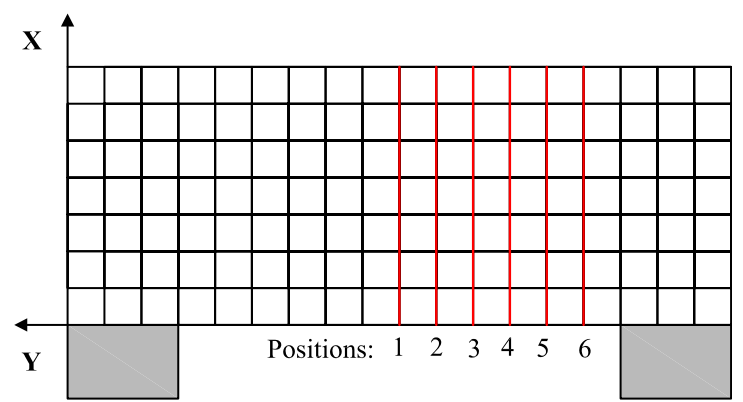

Fig. 6. The locations of six biofilm sections (red lines) in simulation, the negative $Y$-axis is loading direction. 
$X$. Wang et al.

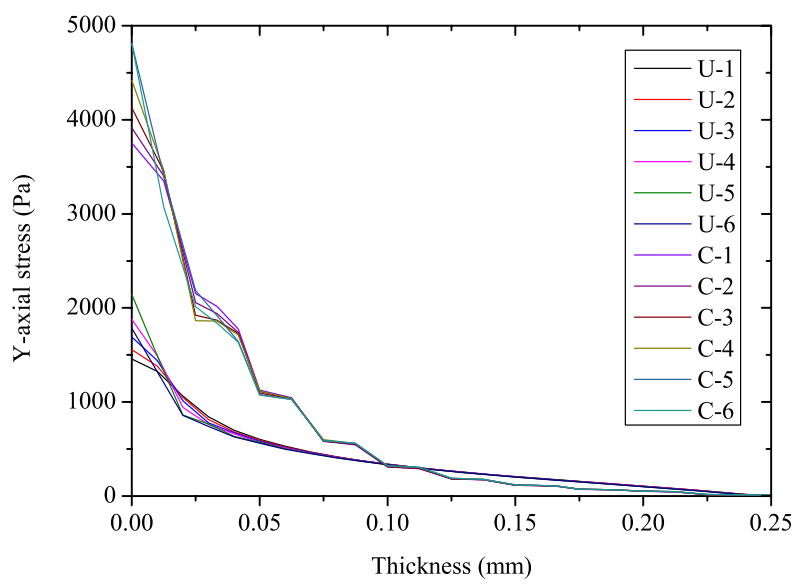

Fig. 7. The $Y$-axial stress curves in two models under displacement loading.

Table 1. The average values of tension stress (in $\mathrm{Pa}$ ) of six paths in two models under displacement loading.

\begin{tabular}{lcccccc}
\hline Positions & 1 & 2 & 3 & 4 & 5 & 6 \\
\hline Composite & 778.1 & 778.6 & 779.5 & 792.7 & 818.2 & 793.3 \\
Uniform & 397.7 & 399.7 & 402.4 & 406.1 & 411.3 & 387.9 \\
\hline
\end{tabular}

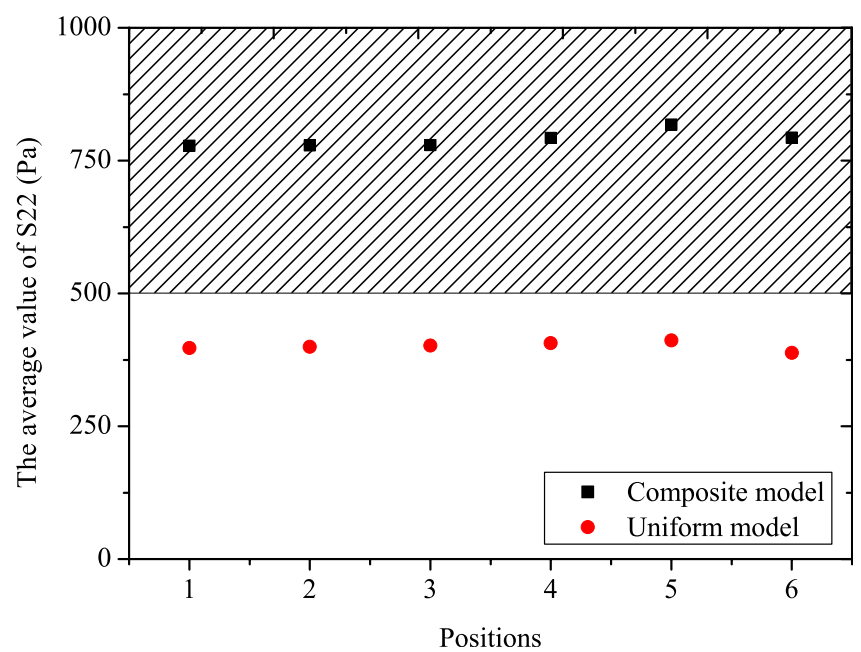

Fig. 8. The average values of normal stress (S22) along the tension direction in two models under displacement loading. The shadow region represents the scope of the experiment measurements (500-1000 Pa). 


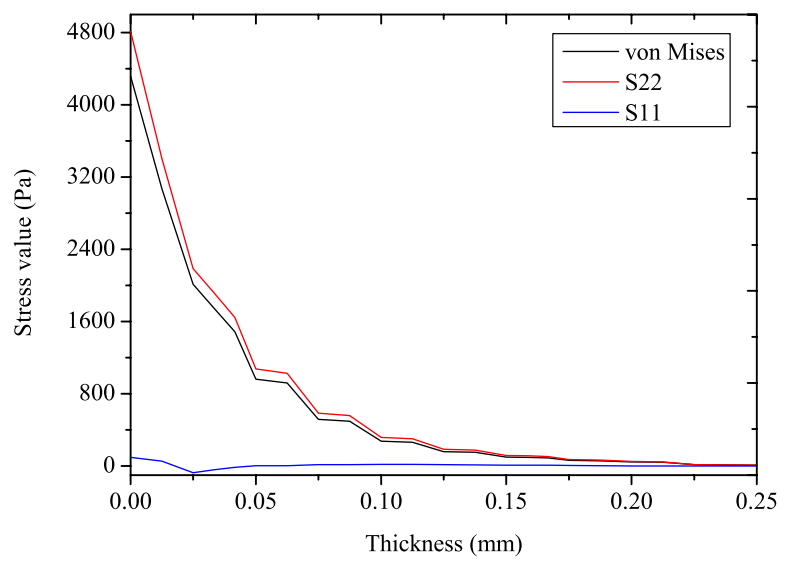

Fig. 9. The values of von Mises stress, normal stress (S22) along the $Y$-axial tension direction, and the normal stress (S11) along the $X$-direction for composite biofilm model under displacement loading.

The stresses near the corner of the tubes are higher than those in the middle of the gap between tubes for both biofilm models, while the stress distribution in composite model is much larger than that in the uniform model. The stress in composite model decreases dramatically along biofilm thickness from $0 \mathrm{~mm}$ to $0.1 \mathrm{~mm}$, as shown in Fig. 7.

We further obtain the values of average tension stress along biofilm thickness in the same regions of biofilm for both models, as shown in Table 1. The average stress in composite biofilm model varies from $778 \mathrm{~Pa}$ to $818 \mathrm{~Pa}$, which is in the range of experimental measurement namely $500-1000 \mathrm{~Pa}$, while the average stress in the uniform biofilm model varies from $387 \mathrm{~Pa}$ to $411 \mathrm{~Pa}$, which do not agree with the experiment measurements, as shown in Fig. 8. Our simulation showed that the composite biofilm model is more appropriate to describe the mechanical properties of biofilms.

In addition, we also obtain von Mises stress, normal stress (S22) which is along $Y$-axial tension direction, and the normal stress (S11) which is along $X$-direction. We find that von Mises stress and the stress along tension direction are almost same, while stress along the other direction is much smaller, as shown in Fig. 9.

\subsection{Force loading condition}

According to experimental measurements, the failure limit of mature biofilm is between $500 \mathrm{~Pa}$ and $1000 \mathrm{~Pa}$ under tension. We choose the average stress of $800 \mathrm{~Pa}$ based on the results from displacement loading, and then obtain the loading force as $F=3.148 \times 10^{-3} \mathrm{~N} .^{16}$

We compare the biofilm extension displacements under force loading condition. Here, we ignore the deformation of polyethylene material, because the Young's modulus of polyethylene material is infinite compared with the biofilm. For the 


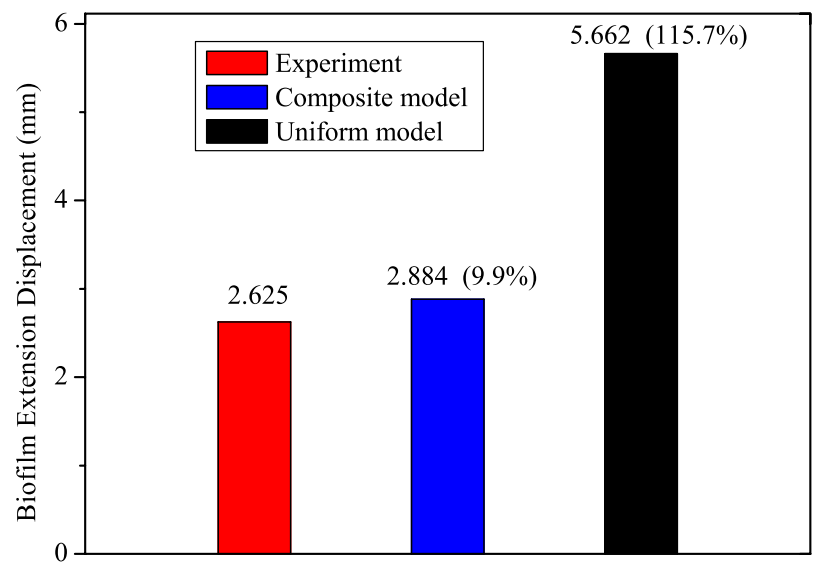

Fig. 10. The uniform biofilm extension displacement (black column), the composite biofilm extension displacement (blue column), and the experiment measurement (red column) under force loading conditions. The differences between different models and the experiment are indicated in brackets.

composite biofilm model, we take the relative displacement between two tubes given directly by ABAQUS as the biofilm extension displacement, which is $2.884 \mathrm{~mm}$, compared to the displacement from experimental prediction, which is $2.625 \mathrm{~mm}$; the difference is $\sigma=|(2.884-2.625) / 2.625| \times 100 \%=9.9 \%$. However, in the uniform biofilm model, the obtained extension displacement of biofilm is $5.662 \mathrm{~mm}$, compared to the displacement from experimental prediction. Here, the difference reaches $\sigma=|(5.662-2.625) / 2.625| \times 100 \%=115.7 \%$, as shown in Fig. 10, which shows that the difference from the uniform biofilm model is larger than the composite biofilm model and it approves that the composite biofilm model is more appropriate than the uniform biofilm model.

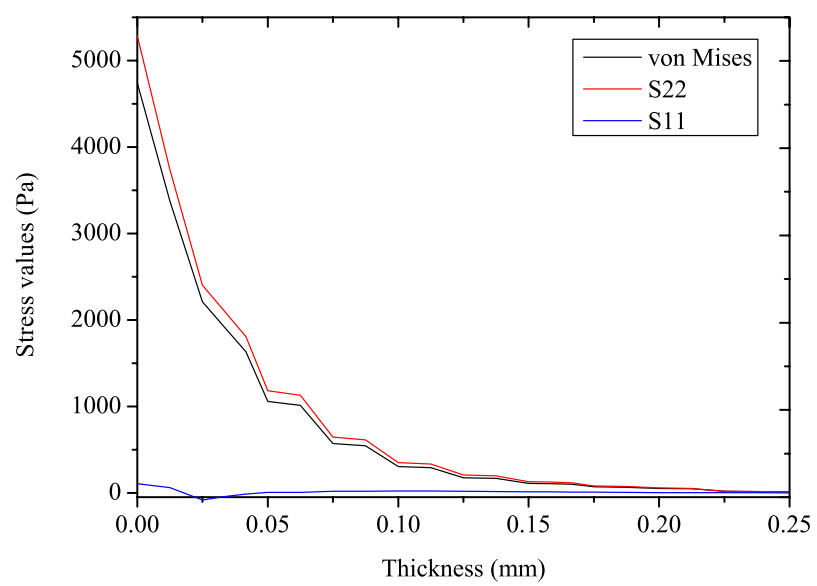

Fig. 11. The values of von Mises stress, normal stress (S22) along the Y-axial tension direction, and the normal stress (S11) along the X-direction in the composite biofilm model under force loading. 
In addition, we can get the stress distribution of von Mises stress, normal stress (S22) which is along the $Y$-axial tension direction, and the normal stress (S11) which is along biofilm thickness direction ( $X$-direction) perpendicular to tension direction. We find that the von Mises stress and stress along the tension direction are almost same, while the stress along other direction is much smaller, as shown in Fig. 11.

\section{Conclusions}

In this paper, we develop a multi-layer composite mechanical model for biofilm; for comparison, we apply both uniform model and our composite model to simulate biofilm tension by using finite element method. We get the following conclusions:

(i) The failure limit from composite mechanical biofilm model agrees well with experimental measurements.

(ii) The force-displacement relationship obtained from composite model of biofilm tension is in accordance with experimental measurements.

(iii) When biofilm is under tension, stress along the tension direction dominated over equivalent stress, and the stress along the other direction is much smaller and can be ignored.

(iv) The stress along the tension direction of composite biofilm model decreases more dramatically than that of uniform biofilm model along biofilm thickness.

Our work provides an alternative theory and finite element simulation procedure to describe mechanical inhomogeneity not only of biofilm but also of other biomaterials.

\section{Acknowledgments}

The work was supported by Harvard University, the National Natural Science Foundation of China (Grant No. 11272002), Opening fund of State Key Laboratory of Nonlinear Mechanics, and Beijing Higher Education Young Elite Teacher Project (Grant No. YETP0363).

\section{References}

1. Vlamakis H, Chai Y, Beauregard P, Losick R, Kolter R, Sticking together: Building a biofilm the Bacillus subtilis way, Nature Rev Microbiol 11:157-168, 2013.

2. Singh R, Paul D, Jain RK, Biofilms: Implications in bioremediation, Trends Microbiol 14:389-397, 2006.

3. Wuertz S, Bishop PL, Wilderer PA, Biofilms in Wastewater Treatment: An Interdisciplinary Approach, IWA Publishing, London, 2003.

4. Gardel EJ, Nielsen ME, Grisdela PT Jr, Girguis PR, Duty cycling influences current generation in multi-anode environmental microbial fuel cells, Environ Sci Technol 46:5222-5229, 2012.

5. Logan BE, Microbial Fuel Cells, John Wiley and Sons, New York, 2008.

6. Fang HH, Xu L-C, Chan K-Y, Effects of toxic metals and chemicals on biofilm and biocorrosion, Water Res 36:4709-4716, 2002. 
X. Wang et al.

7. Yoon SS, Hennigan RF, Hilliard GM, Ochsner UA, Parvatiyar K, Kamani MC, Allen HL, DeKievit TR, Gardner PR, Schwab U, Pseudomonas aeruginosa anaerobic respiration in biofilms: Relationships to cystic fibrosis pathogenesis, Dev Cell 3:593-603, 2002.

8. Anderl JN, Franklin MJ, Stewart PS, Role of antibiotic penetration limitation in Klebsiella pneumoniae biofilm resistance to ampicillin and ciprofloxacin, Antimicrob Agents Chemother 44:1818-1824, 2000.

9. Laspidou C, Spyrou L, Aravas N, Rittmann B, Material modeling of biofilm mechanical properties, Math Biosci 251:11-15, 2014.

10. Dan W-H, Li Z-Q, Liao L-L, Chen M, Zeng R, Dan N-H, Chen C, Lin F, Lin H, Skin collagen and its application in tissue engineering, The Fifth Asian Int Conf Leather Science and Technology, pp. 165-175, 2002.

11. Laspidou CS, Rittmann BE, Evaluating trends in biofilm density using the UMCCA model, Water Res 38:3362-3372, 2004.

12. Beyenal H, Lewandowski Z, Modeling mass transport and microbial activity in stratified biofilms, Chem Eng Sci 60:4337-4348, 2005.

13. Wilking JN, Zaburdaev V, De Volder M, Losick R, Brenner MP, Weitz DA, Liquid transport facilitated by channels in Bacillus subtilis biofilms, Proc Natl Acad Sci USA 110:848-852, 2013.

14. Sinha NN, Visualizing patterns of gene expression in growing Bacillus subtilis biofilms, Ph. D. Thesis, Harvard University, 2013.

15. Wilking J, Angelini T, Seminara A, Brenner M, Weitz D, Biofilms as complex fluids, MRS Bull 36:385-391, 2011.

16. Ohashi A, Koyama T, Syutsubo K, Harada H, A novel method for evaluation of biofilm tensile strength resisting erosion, Water Sci Technol 39:261-268, 1999.

17. Laspidou C, Rittmann BE, Karamanos S, Finite element modeling to expand the UMCCA model to describe biofilm mechanical behavior, Water Sci Technol 52:161-166, 2005.

18. Laspidou CS, Rittmann BE, Modeling the development of biofilm density including active bacteria, inert biomass, and extracellular polymeric substances, Water Res 38:3349-3361, 2004.

19. Willis JR, Elasticity theory of composites, SUDAM Report No. 80-2, Office of the Naval Research, Arlington, VA, USA, 1980.

20. Stoodley P, Lewandowski Z, Boyle JD, Lappin-Scott HM, Structural deformation of bacterial biofilms caused by short-term fluctuations in fluid shear: An in situ investigation of biofilm rheology, Biotechnol Bioeng 65:83-92, 1999.

21. Picioreanu C, van Loosdrecht MC, Heijnen JJ, Two-dimensional model of biofilm detachment caused by internal stress from liquid flow, Biotechnol Bioeng 72:205-218, 2001.

22. Wang X, Koehler SA, Wilking JN, Sinha NN, Cabeen MT, Srinivasan S, Seminara A, Rubinstein S, Sun Q, and Brenner MP. Probing phenotypic growth in expanding Bacillus subtilis biofilms. Applied Microbiology and Biotechnology, 1-9, 2016. 


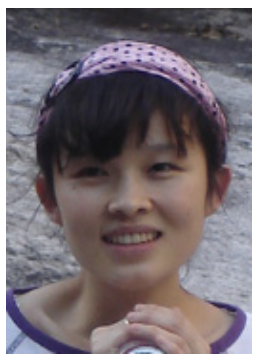

Xiaoling Wang is an Associate Professor at the School of Mechanical Engineering, University of Science and Technology Beijing (USTB), China, and a Visiting Scholar at the Harvard John A. Paulson School of Engineering and Applied Sciences, Harvard University, USA. She received her B.S. and M.S. degrees in Solid Mechanics from Beijing Institute of Technology, China, and a Ph.D. degree in Mechanical Engineering from the Hong Kong University of Science and Technology, Hong Kong. She was a Postdoctoral Fellow at the Hong Kong University, and moved to USTB in 2008. She was a Visiting Scholar in the Northwestern University, USA. Her research interests focus on biomechanics, phase transition in biosystems, and biofilm formation and visualization.

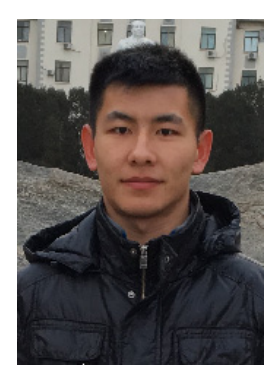

Jingshi Han is pursuing his Master's degree at the School of Mechanical Engineering, USTB, China. He received his B.S. degree in Hebei Institute of Architecture and Civil Engineering, China. His research interest focus on bacterial flagellar filament transition.

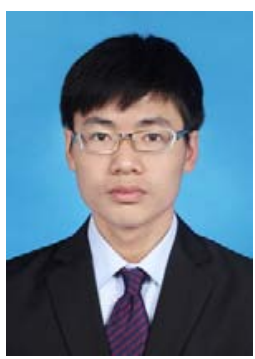

Kui Li received his B.S. degree from the School of Mechanical Engineering, USTB, China. His research interest focus on bacterial flagellar filament transition.

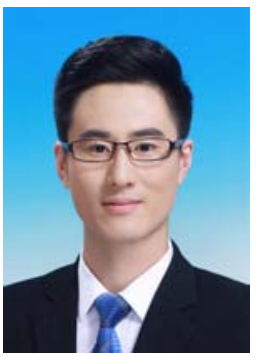

Guoqing Wang is pursuing his Master's degree at the School of Mechanical Engineering, USTB, China. He received his B.S. degree from Weifang University, China. His research interest focus on biofilm growth modeling. 


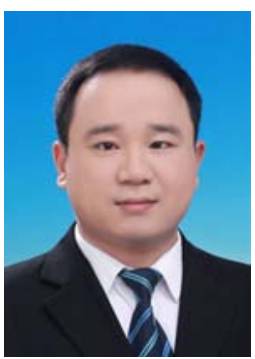

Mudong Hao is pursuing his Master's degree at the School of Mechanical Engineering, USTB, China. He received his B.S. degree from Heze University, China. His research interest focus on biofilm wrinkle mechanics and morphology. 